\title{
Behaviour change in generalised HIV epidemics: impact of reducing cross-generational sex and delaying age at sexual debut
}

\author{
T B Hallett, S Gregson, J J C Lewis, B A Lopman, G P Garnett
}

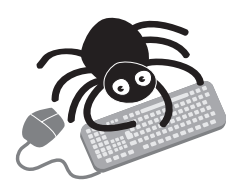

Supplementary material available at http://sti.bmj. com/supplemental

See end of article for authors' affiliations

......................

Correspondence to:

T B Hallett, Department of

Infectious Disease

Epidemiology, Imperial

College London, St Mary's

Campus, Norfolk Place,

London W2 1PG; timothy.

hallett@imperial.ac.uk

Accepted 13 February 2007

Published Online First

21 February 2007

Sex Transm Infect 2007;83(Suppl I):i50-i54. doi: 10.1136/sti.2006.023606

Background: Sexual behavioural change is essential to prevent HIV infections in Africa and statistical analysis of risk factors at the individual-level may be used to design interventions. The importance of reducing crossgenerational sex (young women having sex with older men) and delaying age at first sex on the spread of HIV at the population-level has been presumed but not scientifically investigated and quantified.

Methods: A mathematical model of heterosexual spread of HIV was developed to predict the population-level impact of reducing cross-generational sex and delaying sexual debut.

Results: The impact of behaviour change on the spread of HIV is sensitive to the structure and reaction of the sexual network. Reducing cross-generational sex could have little impact on the risk of infection unless it is accompanied by a reduction in the number of risky sexual contacts. Even peer-to-peer sexual mixing can support high endemic levels of HIV. The benefit of delaying sexual debut is comparatively small and is reduced if males continue to prefer young partners or if young women spend more time unmarried. In Manicaland, Zimbabwe, if older men were to use condoms as frequently as young men, the reduction in risk of infection could exceed that generated by a two-year delay in first sex.

Conclusions: At the individual-level avoiding sex with older partners and delaying sexual debut can decrease the risk of infection but at the population-level these interventions may do little to limit the spread of HIV without wider-ranging behavioural changes throughout the sexual network.
T he HIV pandemic continues to spread devastation globally. ${ }^{1}$ In the generalised epidemics in sub-Saharan Africa, most of the risk is associated with heterosexual sex, ${ }^{2-5}$ and behaviour change is essential to reduce the number of new infections. Funding has been made available for interventions to promote behaviour change, ${ }^{67}$ but care must be taken to target resources at changing those aspects of sexual behaviour that are most important in the spread of HIV.

Observational studies show that usually HIV prevalence among young women far exceeds that among young men. ${ }^{8}$ This has prompted some to emphasise the importance of protecting young women from infection in particular. ${ }^{2-11}$ Statistical analyses of individual behaviour data show that the risk of HIV infection is lower among women who avoid older sexual partners $^{12}{ }^{13}$ and begin sexual activity later. ${ }^{12} 1415$ For this reason, it has been suggested that behaviour interventions should focus on reducing cross-generational sex (young women forming sexual partnerships with older men) and delaying sexual debut. $^{816}{ }^{17}$ The importance of these changes at the population level has been presumed but not scientifically investigated and quantified. ${ }^{8} 111316-20$

Mathematical models allow an assessment of the potential effects of proposed behaviour interventions and can provide a guide to the factors most critical to their success. ${ }^{21}$ Increasingly, the insights gained from mathematical models are well understood and applied, ${ }^{22-24}$ and consulting mathematical models before implementing epidemiological interventions has become standard practice for emerging epidemiological problems. ${ }^{25-27}$ It can be argued that the same approach should be used to assess the effects of proposed behavioural change interventions on generalised HIV epidemics.
In the present study we considered two questions about proposed behavioural change interventions:

- What population-level effect could discouraging cross-generational sex and promoting delayed age at first sex have on the HIV epidemic?

- What properties of the sexual network determine the size of this impact?

To answer these questions we designed a deterministic mathematical model of the heterosexual transmission of HIV with a detailed yearly age structure of young adults so that changes in age at sexual debut, ages of sexual partners and rates of partner change in those recently entering the sexually active population could be investigated. We have parameterised the model using data collected in a cross-sectional survey in rural Zimbabwe ${ }^{12}$ to provide a detailed and well-informed example to illustrate the qualitative results rather than provide an exhaustive description of the various generalised HIV epidemics in Africa.

\section{METHODS}

\section{Data sources}

A stratified population-based survey was carried out in the Manicaland province of eastern Zimbabwe between July 1998 and January 2000. ${ }^{12}$ A follow-up survey was completed after three years. ${ }^{28}$ A structured face-to-face interview was conducted with almost 10000 men and women in 12 distinct communities: two forestry plantations, two tea and coffee estates, two small towns, two roadside trading centres and four subsistence farming areas. In three-quarters of interviews with literate 
respondents, the answers to sensitive questions about sexual behaviour were recorded using a confidential voting method, the use of which has been associated with reporting of greater sexual activity..$^{29}$ Condoms were used in all reported sex acts in the last sexual partnership by $17 \%$ of males and $8 \%$ of females who reported a sexual partnership in the past month. Sex acts were self-defined in the questionnaire, but here we assume that all sex acts are insertive penile-vaginal. The distribution of age difference between sexual partners (male's age minus female's age) takes a similar form for women of all ages and can be modelled by a log-logistic function (figs S1 and S2 in the supplementary material, see http://sti.bmj.com/supplemental). The mean age difference is 7 years, and in approximately $25 \%$ of partnerships the age difference is 10 years or more.

\section{Mathematical simulation model}

We developed a deterministic mathematical model of the heterosexual transmission of HIV in a sex, age and activitystratified population. The model is defined by a set of partial differential equations with respect to time and age that are solved numerically with a four-stage Runge-Kutta algorithm (time-step $=0.02$ years). Full details of the model can be found in the online supplementary material (see http://sti.bmj.com/ supplemental) but the key assumptions in the model are described here.

HIV is assumed to be transmitted through heterosexual partnerships or at the time of birth from an infected mother to her child. To represent the long and variable incubation period and stage-dependent HIV transmission probability, those infected progress through three stages of asymptomatic infection prior to AIDS: the first stage lasts three months and is associated with a high transmission probability; the second stage lasts for several years (up to a mean of 10 years, depending on age) and is associated with a low transmission probability; and the third stage lasts six months and is associated with a high transmission probability.

The population is divided into several sexual activity groups with different numbers of sexual partnerships formed in a year. To permit study of the effect of varying age at first sex and the pattern of partnership formation with respect to age, a detailed yearly age structure was incorporated. On sexual debut most individuals enter the group with the lowest sexual activity, but a few enter groups with much higher sexual activity. An individual of a particular sex, age and sexual activity forms a set number of sexual partnerships each year that are directed preferentially towards those in a similar sexual activity class and between older men and younger women. The fraction of women's partnerships that are formed with men a given number of years older is determined by the log-logistic function. This distribution allows the age difference to be moderate in most sexual partnerships but much greater in others. It can be parameterised so that the pattern of partnership formation matches that observed in Manicaland or varied to represent alternative scenarios (fig S3 in the supplementary material, see http://sti.bmj.com/supplemental). Two types of intervention to reduce cross-generational mixing are simulated:

- a change in the distributional form of the allocation of young women's partnerships among older men, preserving the total number of partnerships formed; and

- removal of a fraction of partnerships with a given age difference, so that there are less partnerships in total.

Both of these interventions generate the same reduction in the fraction of young women's sexual partnerships that are "crossgenerational" but the former replaces those cross-generational partnerships with partnerships among peers whereas the latter does not. For consistency, the derived pattern of partnership formation for one sex must match that of the other, and the extent to which males' demand for sexual partnerships is accommodated by the female population (or vice versa) has to be specified.

Mortality and fertility rates were chosen to represent an African population in the pre-AIDS era and the model was run for 100 years to establish a stable population structure. The infection was introduced to $0.01 \%$ of males aged $25-29$ years in the highest sexual activity group.

\section{RESULTS}

Running the model with the different mixing patterns showed that with more cross-generational mixing, endemic prevalence (among those aged 15-49 years) was slightly higher (fig 1A (bars)). Female-to-male prevalence ratios for 15-24-year-olds at least equal to those observed can be created in the model without assuming any difference in susceptibility to HIV infection between men or women or over age (fig lA (line)).

If all sexual partnerships are formed between peers the epidemic cannot become established (fig 1B). If $1 \%$ of sexual partnerships are formed with partners one year older or younger, HIV can become endemic. Simulating an intervention that reduced cross-generational mixing but preserved the total number of sexual partnership formed, showed that such a behavioural change could substantially reduce HIV prevalence among young women but prevalence among young men might rise (fig $1 \mathrm{C}$ (solid lines)). With this intervention, the lifetime risk of infection (the expected chance at birth that an individual will have been infected with HIV by their fifty-fifth birthday) is reduced by $10 \%$ for females and $5 \%$ for males. In contrast, if the same reduction in cross-generational mixing is simulated but without any compensatory increase in the number of partnerships among peers (dashed lines), prevalence among males can decrease and prevalence among females can decrease further. With this intervention, the lifetime risk of infection is reduced much more: by $25 \%$ for females and $22 \%$ for males.

This pattern of sexual mixing (young women forming partnerships with older men) means that the impact of young women delaying sexual debut is sensitive to the behavioural response of older men-that is, if males' would-be young sexual partners become abstinent, they may seek to replace those last partnerships with the youngest sexually active females available. Females' risk of infection in the first years of sexual activity is therefore linked to whether or not males seek to replace lost partnerships and the extent to which males control the structure of the sexual network (fig 2A). If males do seek to replace the lost partnerships, incidence is increased among women for the first years of sexual activity and this effect is greater if males are able to control the structure sexual network. The reduction in lifetime risk of infection for women is greater if males do not seek to replace lost partnerships (fig 2B). The benefit of delaying sexual debut is even greater if women become less susceptible to infection as they get older. However, in all scenarios the reduction in lifetime risk of infection associated with delaying sexual debut is relatively small.

Another issue is the relative timing of sexual debut and marriage. ${ }^{30}$ The risk of infection when sexually active but not married ( $2 \%$ per person-year at risk during the first three years of follow-up in this population) is greater than when married ( $1 \%$ per person-year). This means that the benefit of delaying first sex depends on the time spent unmarried. For example, increasing the age at first sex from 18 to 21 could lead to lifetime risk of infection being reduced by $9 \%$ (if women are unmarried for 12 months less), reduced by 6\% (no change in relative timing of marriage) or remain almost unchanged (if women are unmarried for an extra three years). 
A

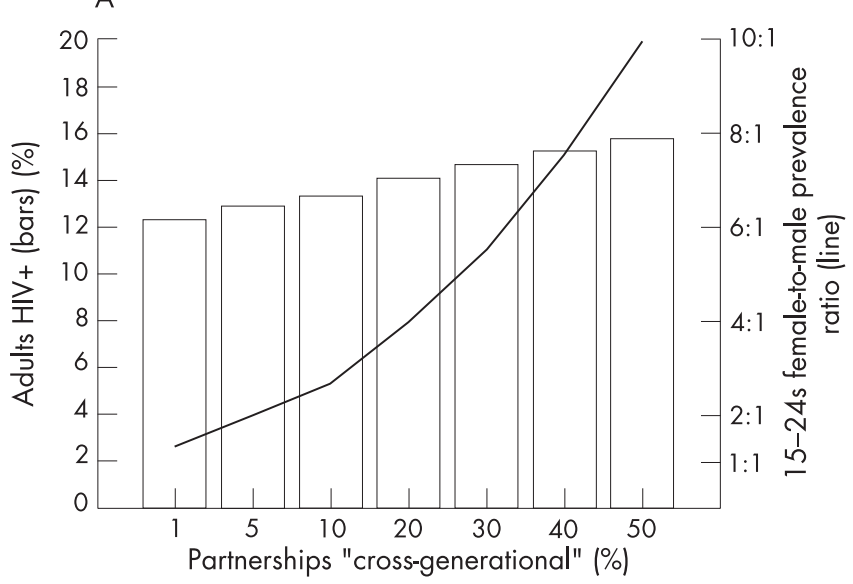

B

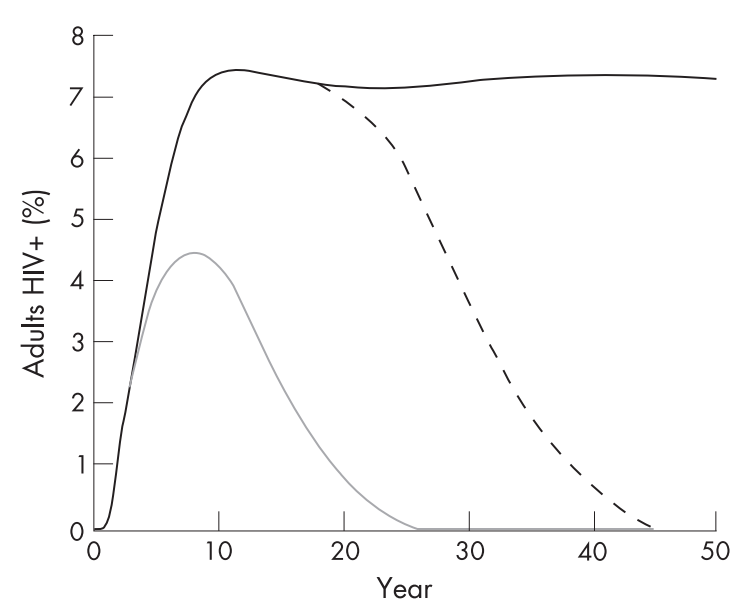

C

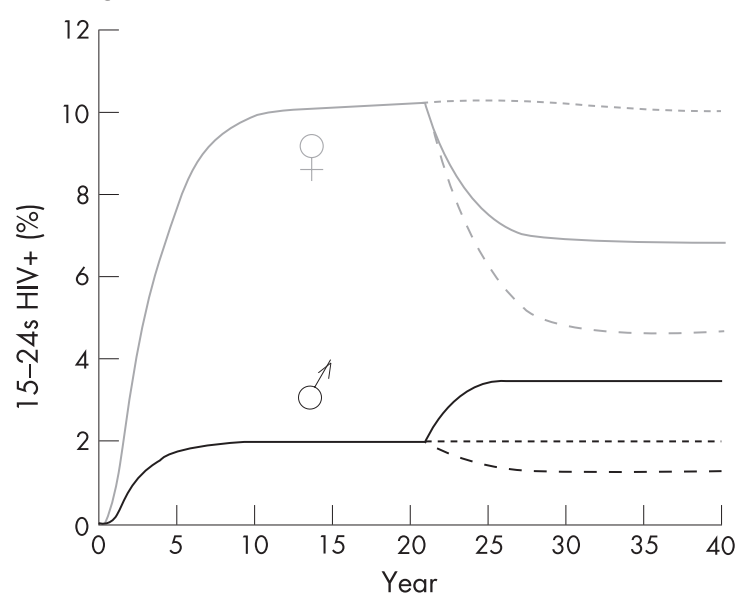

Figure 1 The impact of reducing cross-generational sex on the heterosexual spread of HIV. (A) HIV prevalence among 15-49-year-olds (bars) and female-to-male prevalence among 15-24-year-olds (line) with different levels of cross-generational mixing (percent of females' partnerships formed with males 10 or more years older), 20 years after infection introduced. (B) HIV prevalence over time if all partnerships are formed between individuals the same age (grey line) or if $1 \%$ of partnerships are formed between individuals one year older or younger (black line), or if an intervention eliminates any non-peer-to-peer partnerships at year 20 (dashed line). (C) HIV prevalence over time for 1524-year-old males (black) and females (grey) if the proportion of crossgenerational partnerships is reduced from $20 \%$ to $5 \%$ at year 20 and if there is replacement with peer-to-peer partnerships (solid lines) or if there is no replacement (dashed lines); dotted lines show "no change" scenario.

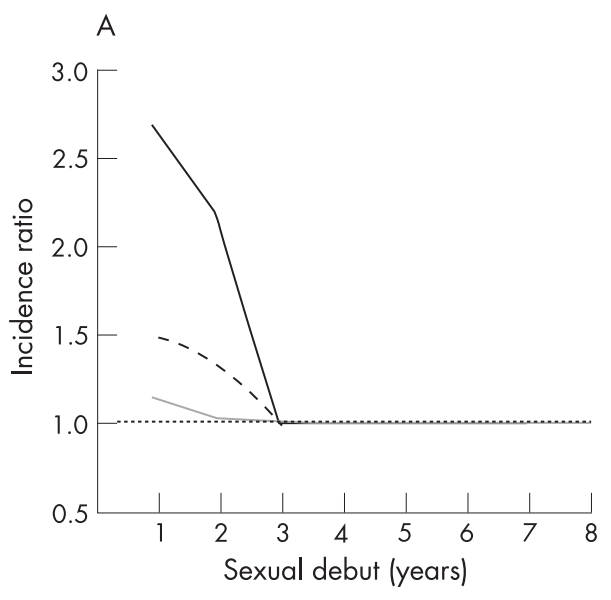

B

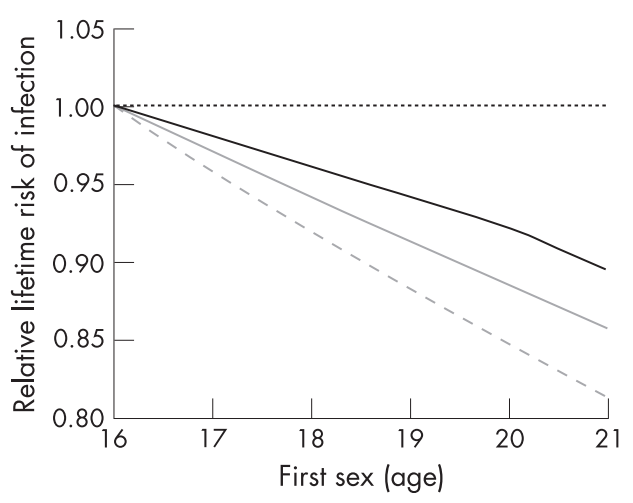

Figure 2 The impact of delaying age at first sex on the heterosexual spread of HIV. (A) HIV incidence ratio for women when age at first sex is delayed by two years for both sexes if males seek to maintain their current level of sexual activity and if mixing is determined by males (black line), females (grey line) or is an equal compromise (dashed line). (B) Relative lifetime risk of infection when age at first sex is increased from 16 years if males seek to maintain their number of sexual partnerships (black line) or if partnerships are not replaced (grey line) or if partnerships are not replaced and susceptibility to infection decreases with age (dashed line).

In rural Zimbabwe, the probability of reporting having used a condom consistently with their most recent sexual partner is particularly low for older men (25 years or older) but is higher if either the man or his partner has had many sexual partners (fig 3A). If condom use by older men is increased to the level currently reported by younger men, the lifetime risk of infection could be reduced by $\sim 20 \%$ for both males and females. This exceeds the impact of doubling condom use in the whole population or males and females delaying sexual debut by two years under the most optimistic assumptions (fig 3B).

\section{DISCUSSION}

Sexual behaviour change is crucial to prevent HIV infections in sub-Saharan Africa. ${ }^{2}$ Cross-sectional surveys of individuals' self-reported sexual behaviour show that young women who have had sex with an older man or begun sexual activity when young are more likely to have been infected with HIV. ${ }^{12-15}$ On this basis, interventions aiming to change behaviour have been proposed that focus on discouraging cross-generational sex and delaying sexual debut. ${ }^{816}$ Although it is clear that these changes will somewhat reduce the risk of infection to the individual it was not known what impact they could have on the spread of HIV through the population. Despite this uncertainty the importance of cross-generational sex in supporting high HIV prevalence has been presumed, ${ }^{17}$ and an increase in abstinence among young people has been partially 

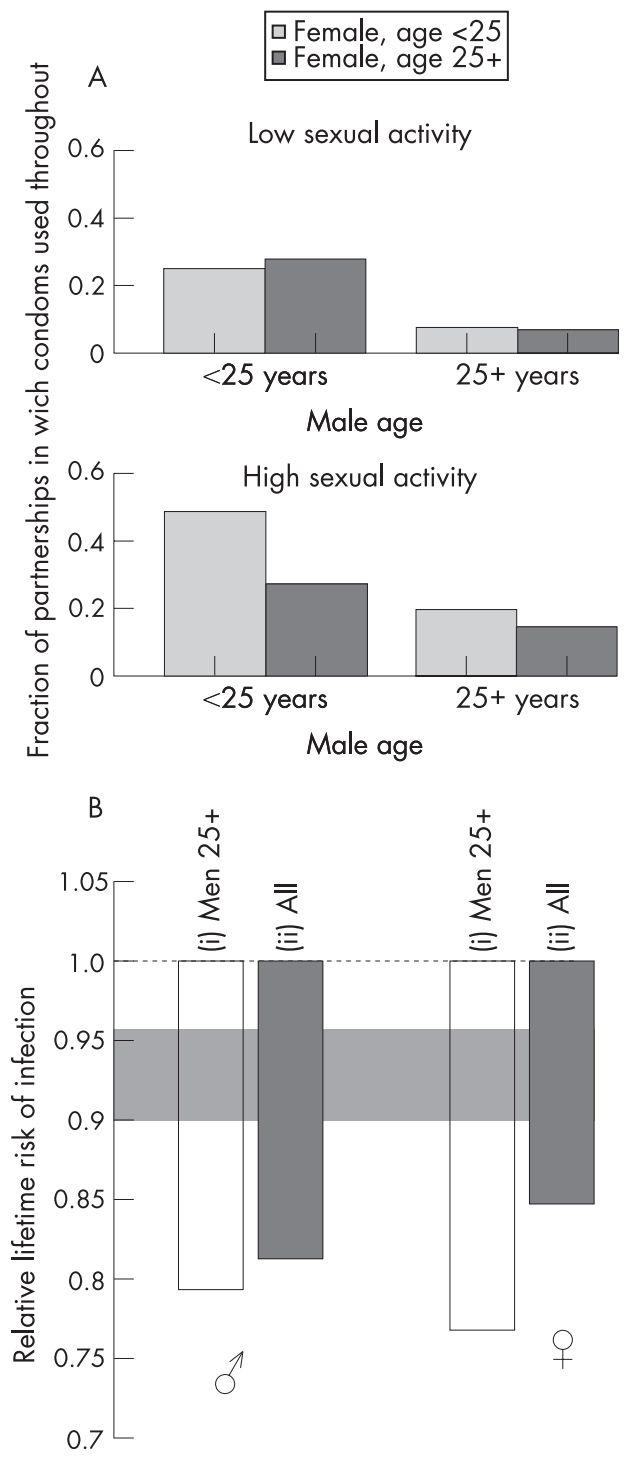

Figure 3 The impact of increasing condom use on the heterosexual spread of HIV. (A) The proportion of reported partnerships in which condoms were used in all sex acts, by the age of each partner and whether either one of the partners reported having had six or more previous sexual partners (highly sexually active). (B) Relative lifetime risk of infection for males and females if (1) condom use among males older than 25 increases to the level reported by males under 25 (open bars), (2) condom use overall is doubled (grey bars). The horizontal grey shading gives the comparable range of effects that could be expected if age at first sex for males and females is increased by two years.

credited with the decline in HIV prevalence rates in Uganda. ${ }^{31}$ In this study we used a mathematical model to quantify the reduction in the average lifetime risk of HIV infection that reducing cross-generational sex and delaying first sex could bring. The model was carefully parameterised using detailed data from a rural district in eastern Zimbabwe ${ }^{12}$ and we expect that the qualitative insights will be generalisable to other African settings with epidemics.

Our model simulations suggest that HIV prevalence is only slightly higher with much more cross-generational mixing. Furthermore, even if $99 \%$ of sexual partnerships are formed between peers, HIV can still reach a high endemic level. This is contrary to the suggestion that without cross-generational mixing HIV could not persist endemically. ${ }^{17}$ Cross-generational sex helps increase prevalence because few of the partnerships of infected older males are with older females who are already
Key messages

- The impact of behaviour change on HIV spread is sensitive to the structure and reaction of the sexual network.

- Reducing cross-generational sex may have little effect on the risk of infection unless it is accompanied by a reduction in the number of risky sexual contacts.

- The benefit of delaying age at sexual debut is comparatively small and is reduced if males continue to prefer young partners or if young women spend more time unmarried.

infected too and more will probably be with uninfected younger females. However, the link is not strong because the infection will reach all age groups eventually under almost any mixing scenario, and then young women will be exposed instead to the high rates of sexual activity of their male peers. Sex with older men is more risky than sex with younger men partly because of the greater possibility of the former to be infected with HIV, having been exposed to the risk of infection for longer. However, the observed gradient in HIV prevalence among males by age is partly a result of the cross-generational mixing; prevalence among young men is suppressed by cross-generational mixing because they have only limited access to their peers and instead have sex with even younger uninfected women or not at all. A switch to peer-to-peer mixing will increase prevalence among young men making, from a female perspective, formerly "low-risk" contacts increasingly "high risk". In this way, the benefit of peer-to-peer over crossgenerational mixing to the young women is negatively frequency dependent; one young woman will put herself at less risk by choosing partners her own age over older men but as more women come to do the same the benefit of this behaviour change is reduced as HIV prevalence among young men increases.

The real impact of cross-generational sex on the populationlevel spread of HIV is the power imbalance in those partnerships and the implications this has for the chance that condoms will be used. ${ }^{12}{ }^{19}$ Our results show that behaviour change interventions should aim to minimise unprotected sexual contact by young women with anyone and should not target the age difference between sexual partners per se. Nonetheless, this pattern of partnership formation between older men and younger women does seem to underlie the high female-to-male HIV prevalence ratio among young people; the model generates ratios at least as extreme as those observed without assuming any difference in susceptibility to infection by sex or age. Crossgenerational mixing must be considered when examining other types of behavioural intervention; the demand for their sexual partnerships could undermine the benefit young women get from remaining abstinent for longer and the importance of transmission from older men makes the frequency with which they use condoms especially important.

Abstinence (through delaying the onset of sexual activity) removes the risk of infection for only a few years-a small fraction of one's lifetime exposure. This period may be important because the first years of a woman's sexual activity may carry the greatest risk of transmission for a number of biological $^{33-35}$ and behavioural ${ }^{12}{ }^{18}$ reasons. However, even assuming that susceptibility declines strongly with age, the lifetime benefit of delaying sex for two years is expected to be, at most, only $8 \%$. The benefit is smaller (3\%) if the "demand" for their sexual partnerships is not reduced. Although 
abstinence undeniably reduces the risk of infection, its impact on the spread of HIV and the average chance of infection over a lifetime is small. It is probable, therefore, that the dramatic prevalence decline in Uganda ${ }^{31}$ was primarily mediated through increased condom use and fidelity rather than the two-year delay in first sex that was also reported. ${ }^{32}$

In the coming years, ongoing and proposed behaviour interventions will be assessed and their potential effect estimated. This modelling work has highlighted the sensitivity of the efficacy of some behaviour changes to unknown properties of the sexual network (eg the response of males to female behaviour change) and projections should incorporate this uncertainty.

From an individual-level perspective it is possible to identify those elements of sexual behaviour that are associated with the highest risk of infection. However, without a dynamic description of HIV transmission it is not clear how these changes will limit the spread of HIV at the population level. The chance of infection is only partly determined by one's own sexual behaviour and interventions should not be narrowly targeted at particular at-risk groups (for example, young women) but instead should tackle risky behaviour throughout the sexual network.

\section{ACKNOWLEDGEMENTS}

TBH, SG, JJCL and BAL thank the Wellcome Trust, and GPG thanks the UK Medical Research Council for funding support.

\section{AUTHOR CONTRIBUTIONS}

All authors conceived the study, assisted with model design and analysis, and drafted the manuscript.

\section{Authors' affiliations}

T B Hallett, S Gregson, B A Lopman, G P Garnett, Department of Infectious Disease Epidemiology, Imperial College London, London UK S Gregson, Biomedical Research and Training Institute, Zimbabwe J J C Lewis, London School of Hygiene and Tropical Medicine, London, UK; Aurum Institute for Health Research, Johannesburg, South Africa

Competing interests: None.

\section{REFERENCES}

1 UNAIDS/WHO. AIDS epidemic update: December 2005. Geneva: UNAIDS/ WHO, 2005

2 UNAIDS. 2004 Report of the global AIDS epidemic. Geneva: UNAIDS, 2004

3 Kiwanuka N, Gray RH, Serwadda D, et al. The incidence of HIV-1 associated with injections and transfusions in a prospective cohort, Rakai, Uganda. AIDS 2004; 18:342-4.

4 Schmid GP, Buve A, Mugyenyi P, et al. Transmission of HIV-1 infection in subSaharan Africa and effect of elimination of unsafe injections. Lancet 2004;363:482-8.

5 Lopman BA, Garnett GP, Mason PR, et al. Individual level injection history: a lack of association with HIV incidence in rural Zimbabwe. PLoS Med 2005:e37.

6 Office of the United States Global AIDS Coordinator. The President's Emergency Plan for AIDS Relief. US Five-year global HIV/AIDS strategy, 2004. Washington DC: Office of the Global AIDS Coordinator, 2004.

7 The Global Fund to fight AIDS, tuberculosis and malaria. Geneva: The Global Fund to Fight AIDS, Tuberculosis and Malaria, 2004.
8 Laga M, Schwartlander B, Pisani E, et al. To stem HIV in Africa, prevent transmission to young women. AIDS 2001;15:931-4.

9 Genuis SJ, Genuis SK. Adolescent behaviour should be priority. BMJ 2004;328:894.

10 Green EC, Berman J. Liaisons fueling AIDS in Africa. The Washington Times. 28 December, 2003

11 AIDSMark. Cross-generational sex fueling the HIV/AIDS epidemic in subSaharan Africa, International Centre for Research on Women (ICRW) and Population Services International (PSI), 2003.

12 Gregson S, Nyamukapa CA, Garnett GP, et al. Sexual mixing patterns and sexdifferentials in teenage exposure to HIV infection in rural Zimbabwe. Lancet 2002;359:1896-903.

13 Kelly RJ, Gray RH, Sewankambo NK, et al. Age differences in sexual partners and risk of HIV-1 infection in rural Uganda. J Acquir Immune Defic Syndr 2003;32:446-51.

14 Pettifor AE, Van Der Straten A, Dunbar MS, et al. Early age of first sex: a risk factor for HIV infection among women in Zimbabwe. AIDS 2004; 18:1435-42.

15 Drain PK, Smith JS, Hughes JP, et al. Correlates of national HIV seroprevalence: an ecologic analysis of 122 developing countries. J Acquir Immune Defic Syndr 2004;35:407-20.

16 Population Services International. Dangerous Liaisons, Research Brief No. 2, Washington DC: PSI, 2003.

17 Cohen J. Asia and Africa: on different trajectories? Science 2004;304:1932-8.

18 Longfield K, Glick A, Waithaka M, et al. Relationships between older men and younger women: implications for STIs/HIV in Kenya. Stud Fam Plann 2004; $35: 125-34$

19 Luke N, Kurz KM. Cross-generational and transactional sexual relationships in Sub-Saharan Africa. Washington DC: International Centre for Research on Women, 2002

20 Buve A, Bishikwabo-Nsarhaza K, Mutangadura G. The spread and effect of HIV1 infection in sub-Saharan Africa. Lancet 2002;359:201 1-7.

21 Grassly NC, Garnett GP, Schwartlander B, et al. The effectiveness of HIV prevention and the epidemiological context. Bull World Health Organ 2001;79:1121-32.

22 Anderson RM, Garnett GP. Mathematical models of the transmission and control of sexually transmitted diseases. Sex Transm Dis 2000;27:636-43.

23 Garnett GP. An introduction to mathematical models in sexually transmitted disease epidemiology. Sex Transm Infect 2002;78:7-12.

24 Aral SO, Roegner R. Mathematical modeling as a tool in STD prevention and control: a decade of progress, a millennium of opportunities. Sex Transm Dis 2000;27:556-7

25 Keeling MJ, Woolhouse ME, Shaw DJ, et al. Dynamics of the 2001 UK foot and mouth epidemic: stochastic dispersal in a heterogeneous landscape. Science $2001 ; 294: 813-7$

26 Riley S, Fraser C, Donnelly CA, et al. Transmission dynamics of the etiological agent of SARS in Hong Kong: impact of public health interventions. Science 2003;300:1961-6.

27 Ferguson NM, Cummings DA, Cauchemez S, et al. Strategies for containing an emerging influenza pandemic in Southeast Asia. Nature 2005;437:209-14.

28 Gregson S, Garnett GP, Nyamukapa CA, et al. HIV decline associated with behavior change in eastern Zimbabwe. Science 2006;311:664-6.

29 Gregson S, Zhuwau T, Ndlovu J, et al. Methods to reduce social desirability bias in sex surveys in low-development settings: experience in Zimbabwe. Sex Transm Dis 2002;29:568-75.

30 Zaba B, Pisani E, Slaymaker E, et al. Age at first sex: understanding recent trends in African demographic surveys. Sex Transm Infect 2004;80(Suppl 2):ii28-35.

31 Stoneburner RL, Low-Beer D. Population-level HIV declines and behavioral risk avoidance in Uganda. Science 2004;304:714-8.

32 Asiimwe-Okiror G, Opio AA, Musinguzi J, et al. Change in sexual behaviour and decline in HIV infection among young pregnant women in urban Uganda. AIDS 1997;11:1757-63.

33 Glynn JR, Carael M, Auvert B, et al. Why do young women have a much higher prevalence of HIV than young men? A study in Kisumu, Kenya and Ndola, Zambia. AIDS 2001;15(Suppl 4):S51-60.

34 Moss GB, Clemetson D, D'Costa L, et al. Association of cervical ectopy with heterosexual transmission of human immunodeficiency virus: results of a study of couples in Nairobi, Kenya. J Infect Dis 1991;164:588-91.

35 Duncan ME, Tibaux G, Pelzer A, et al. First coitus before menarche and risk of sexually transmitted disease. Lancet 1990;335:338-40. 